\title{
Quantum-Mechanical Dualities from Classical Phase Space
}

\author{
José M. Isidro \\ Instituto de Física Corpuscular (CSIC-UVEG) \\ Apartado de Correos 22085, Valencia 46071, Spain \\ jmisidro@ific.uv.es
}

October 30, 2018

\begin{abstract}
The geometry of the classical phase space $\mathcal{C}$ of a finite number of degrees of freedom determines the possible duality symmetries of the corresponding quantum mechanics. Under duality we understand the relativity of the notion of a quantum with respect to an observer on $\mathcal{C}$. We illustrate this property explicitly in the case when classical phase space is complex $n$-dimensional projective space $\mathbf{C P}^{n}$. We also provide some examples of classical dynamics on $\mathbf{C P}^{n}$ that exhibit these properties at the quantum level.

Keywords: Classical phase space; duality.

2001 PACS codes: 03.65.Bz, 03.65.-w, 03.65.Ca, 03.65.Sq.
\end{abstract}

\section{Contents}

1 Introduction 1

2 Ouantum Hilbert-space bundles over $\mathbf{C P}^{n} \quad 3$

2.1 Construction of the bundle $\mathcal{Q H} \mathcal{H}_{-1}\left(\mathbf{C P}^{n}\right) \ldots \ldots$. . . . . . . . 3

2.2 Diagonalisation of the projective Hamiltonian . . . . . . . . . . 4

2.3 Construction of the bundle $\mathcal{Q H}\left(\mathcal{H}_{(1), 1)}\left(\mathbf{C P}^{n}\right) \ldots \ldots\right.$

$\begin{array}{lll}3 & \text { Discussion } & 7\end{array}$

\section{Introduction}

Duality can be understood as the relativity of the notion of a quantum [1]. Following ref. [1], a framework is needed that can implement dualities in the quantum mechanics of a finite number of degrees of freedom [2]. The purpose of this letter is to expand on previous work [3] 4], where such a framework has been presented. We would also like to draw attention to refs. [5, 6, 7]. 
The notion of duality implies the possibility of nontrivial transformations between the Hilbert spaces of quantum states corresponding to different observers on classical phase space $\mathcal{C}$. Under an observer one understands, in general relativity, a little man carrying a ruler and a clock. In fact one may forget about the little man while keeping his ruler and his clock, to conclude that an observer is just a local coordinate chart on spacetime. (We adopt the active point of view, under which a coordinate change corresponds to a transformation between different spacetime points). Mutatis mutandi, in a quantum-mechanical setup, an observer will be a local coordinate chart $(\mathcal{U}, z)$ on $\mathcal{C}$, where $\mathcal{U}$ is an open neighbourhood of a point $p \in \mathcal{C}$ endowed with the coordinate functions $z$. We intend to develop the quantum theory corresponding to this observer. Pick a vacuum state $|0(p)\rangle$ (obtained by minimising a certain Hamiltonian on $\mathcal{U}$ ). Under a coordinate change from $p$ to $\tilde{p}$ we have $(\mathcal{U}, z) \rightarrow(\tilde{\mathcal{U}}, \tilde{z})$, and the vacuum changes as $|0(p)\rangle \rightarrow|0(\tilde{p})\rangle$. Performing this operation sufficiently many times we succeed in covering $\mathcal{C}$ with a family of 1 -dimensional vector spaces, pointwise generated by the corresponding vacua. Geometrically we have defined a complex line bundle $N(\mathcal{C})$ over $\mathcal{C}$, whose fibre over $p \in \mathcal{C}$ is generated by $|0(p)\rangle$.

Corresponding to $N(\mathcal{C})$ we can erect a Hilbert-space fibre $\mathcal{H}(p)$ over $p \in \mathcal{C}$ by considering all possible quantum excitations of $|0(p)\rangle$. Letting then $p$ vary over $\mathcal{C}$, we define a complex vector bundle $\mathcal{Q H}(\mathcal{C})$ that we call quantum Hilbert-space bundle, or just $\mathcal{Q H}$-bundle for short.

Now it is clear that different observers on $\mathcal{C}$ may be quantised differently, even after a fixed choice for the vacuum line bundle $N(\mathcal{C})$. If $\mathcal{Q H}(\mathcal{C})$ is a flat bundle, parallel transport allows to canonically identify the fibres $\mathcal{H}(p), \mathcal{H}(\tilde{p})$ over different points. (The connection itself will be univocally defined by requiring it to be metriccompatible and torsion-free). However, on a nonflat bundle there is no such canonical identification between different fibres; there is also no a priori reason for $\mathcal{Q H}(\mathcal{C})$ to be flat. Only on certain manifolds $\mathcal{C}$ can we ensure that $\mathcal{Q H}(\mathcal{C})$ will be flat $($ e.g., when $\mathcal{C}$ is contractible). In the general case $\mathcal{Q H}(\mathcal{C})$ will be nonflat. The absence of a canonical identification between the fibres above different points allows for duality transformations. For example, one such imaginable duality could be having a semiclassical state $|\psi(p)\rangle \in \mathcal{H}(p)$ transform into a highly quantum excitation $|\psi(\tilde{p})\rangle \in \mathcal{H}(\tilde{p})$ when passing from $p$ to $\tilde{p}$. The precise nature of the specific dualities so obtained will depend on the geometry of $\mathcal{C}$ and $\mathcal{Q H}(\mathcal{C})$.

Another possible implication of duality is the following. Quantum excitations may be measured with respect to different vacua. The corresponding quanta are physically inequivalent. Allowing for more than one, physically inequivalent, vacuum is possible if $\mathcal{C}$ admits more than one equivalence class of complex line bundles $N(\mathcal{C})$.

Our notations are as follows (see refs. [8 9] 10] for background material). $\mathcal{C}$ will denote a complex $n$-dimensional, compact classical phase space, endowed with a symplectic form $\omega$ and a complex structure $\mathcal{J}$. We will assume that $\omega$ and $\mathcal{J}$ are compatible, so holomorphic coordinate charts on $\mathcal{C}$ are also Darboux charts. On a complex manifold $\mathcal{C}$, the Picard group $\operatorname{Pic}(\mathcal{C})$ classifies holomorphic equivalence classes of complex line bundles $N(\mathcal{C})$.

We will concentrate on the case when $\mathcal{C}$ is complex projective space $\mathbf{C} \mathbf{P}^{n}$. Then we have $\operatorname{Pic}\left(\mathbf{C P}^{n}\right)=\mathbf{Z}$. In this way inequivalent, holomorphic line bundles $N_{l}\left(\mathbf{C P}^{n}\right)$ over $\mathbf{C P}^{n}$ are 1-to-1 with the integers $l \in \mathbf{Z}$. The following holomorphic vector 
bundles over $\mathbf{C P}^{n}$ will be considered: the hyperplane bundle $\tau$ and its dual $\tau^{*}$, also called the tautological line bundle; the holomorphic tangent bundle $T\left(\mathbf{C P}^{n}\right)$ and its dual $T^{*}\left(\mathbf{C P}^{n}\right)$. Every holomorphic line bundle $N_{l}\left(\mathbf{C P}^{n}\right)$ over $\mathbf{C} \mathbf{P}^{n}$ is isomorphic to $\tau^{l}$ for some $l \in \mathbf{Z}$; this integer is the Picard class. The fibrewise generator of $N_{l}\left(\mathbf{C P}^{n}\right)$ is the vacuum state $|0\rangle_{l}$. Compactness of $\mathbf{C P}^{n}$ implies that the Hilbert space $\mathcal{H}$ is finite-dimensional; we will show that the minimum value of $\operatorname{dim} \mathcal{H}$ is $n+1$.

\section{Quantum Hilbert-space bundles over $\mathrm{CP}^{n}$}

\subsection{Construction of the bundle $\mathcal{Q H}_{l=1}\left(\mathbf{C P}^{n}\right)$}

Let $Z^{1}, \ldots, Z^{n+1}$ denote homogeneous coordinates on $\mathbf{C P}^{n}$. The chart defined by $Z^{k} \neq 0$ covers one copy of the open set $\mathcal{U}_{k}=\mathbf{C}^{n}$. On the latter we have the holomorphic coordinates $z_{(k)}^{j}=Z^{j} / Z^{k}, j \neq k$; there are $n+1$ such coordinate charts.

To begin with we will choose the Picard class $l=1$. Starting from $\mathcal{C}=\mathbf{C P}^{0}$, i.e., a point $p$ as classical phase space, the space of quantum rays must also reduce to a point. Then the corresponding Hilbert space is $\mathbf{C}$. The only state is the vacuum $|0\rangle_{l=1}$.

Next we pass from $\mathcal{C}=\mathbf{C P}^{0}$ to $\mathcal{C}=\mathbf{C P}^{1}$. Regard $p$, henceforth denoted $p_{1}$, as the point at infinity with respect to a coordinate chart $\left(\mathcal{U}_{1}, z_{(1)}\right)$ on $\mathbf{C} \mathbf{P}^{1}$ that does not contain $p_{1}$. This chart is biholomorphic to $\mathbf{C}$ and supports a representation of the Heisenberg algebra in terms of creation and annihilation operators $A^{\dagger}(1), A(1)$. This process adds the new state $A^{\dagger}(1)|0\rangle_{l=1}$ to the spectrum. The new Hilbert space $\mathbf{C}^{2}$ is the linear span of $|0\rangle_{l=1}$ and $A^{\dagger}(1)|0\rangle_{l=1}$.

On $\mathbf{C P}^{1}$ we have the charts $\left(\mathcal{U}_{1}, z_{(1)}\right)$ and $\left(\mathcal{U}_{2}, z_{(2)}\right)$. Point $p_{1}$ is at infinity with respect to $\left(\mathcal{U}_{1}, z_{(1)}\right)$, while it belongs to $\left(\mathcal{U}_{2}, z_{(2)}\right)$. Similarly, the point at infinity with respect to $\left(\mathcal{U}_{2}, z_{(2)}\right)$, call it $p_{2}$, belongs to $\left(\mathcal{U}_{1}, z_{(1)}\right)$ but not to $\left(\mathcal{U}_{2}, z_{(2)}\right)$. Above we have proved that the $\mathcal{Q H}$-bundle has a fibre $\mathbf{C}^{2}$ which, on the chart $\mathcal{U}_{1}$, is the linear span of $|0\rangle_{l=1}$ and $A^{\dagger}(1)|0\rangle_{l=1}$. On the chart $\mathcal{U}_{2}$, the fibre is the linear span of $|0\rangle_{l=1}$ and $A^{\dagger}(2)|0\rangle_{l=1}, A^{\dagger}(2)$ being the creation operator on $\mathcal{U}_{2}$. On the common overlap $\mathcal{U}_{1} \cap \mathcal{U}_{2}$, the coordinate transformation between $z_{(1)}$ and $z_{(2)}$ is holomorphic. This implies that, on $\mathcal{U}_{1} \cap \mathcal{U}_{2}$, the fibre $\mathbf{C}^{2}$ can be taken in either of two equivalent ways: either as the linear span of $|0\rangle_{l=1}$ and $A^{\dagger}(1)|0\rangle_{l=1}$, or as that of $|0\rangle_{l=1}$ and $A^{\dagger}(2)|0\rangle_{l=1}$.

The general construction is now clear. Topologically we have $\mathbf{C P}^{n}=\mathbf{C}^{n} \cup$ $\mathbf{C P}^{n-1}$, with $\mathbf{C P}^{n-1}$ a hyperplane at infinity, but we also need to describe the coordinate charts and their overlaps. There are coordinate charts $\left(\mathcal{U}_{k}, z_{(k)}\right), k=1, \ldots, n+1$ and nonempty $f$-fold overlaps $\cap_{k=1}^{f} \mathcal{U}_{k}$ for $f=2,3, \ldots, n+1$. Each chart $\left(\mathcal{U}_{k}, z_{(k)}\right)$ is biholomorphic with $\mathbf{C}^{n}$ and has a $\mathbf{C P}^{n-1}$-hyperplane at infinity; the latter is charted by the remaining charts $\left(\mathcal{U}_{r}, z_{(r)}\right), r \neq k$. Over $\left(\mathcal{U}_{k}, z_{(k)}\right)$ the Hilbert-space bundle $\mathcal{Q H}$ has a fibre $\mathbf{C}^{n+1}$ spanned by

$$
|0\rangle_{l=1}, \quad A_{i}^{\dagger}(k)|0\rangle_{l=1}, \quad i=1,2, \ldots, n .
$$

Analyticity arguments similar to those above prove that, on every nonempty $f$-fold overlap $\cap_{k=1}^{f} \mathcal{U}_{k}$, the fibre $\mathbf{C}^{n+1}$ can be taken in $f$ different, but equivalent ways, as the linear span of $|0\rangle_{l=1}$ and $A_{i}^{\dagger}(k)|0\rangle_{l=1}, i=1,2, \ldots, n$, for every choice of $k=$ $1, \ldots, f$. 
For the transition functions we take the following. The vacuum state $|0\rangle_{l=1}$ transforms with the transition function $t(\tau)$ corresponding to the line bundle $N_{l=1}\left(\mathbf{C P}^{n}\right)=$ $\tau$. The excited states $A_{i}^{\dagger}(k)|0\rangle_{l=1}$ will transform according to $n \times n$ jacobian matrices $j\left(\mathbf{C P}^{n}\right)$ for the coordinate changes on $\mathbf{C P}^{n}$. In this way the $A_{i}^{\dagger}(k)|0\rangle_{l=1}$ provide (fibrewise) a basis for the tangent bundle $T\left(\mathbf{C P}^{n}\right)$. Altogether, the transition functions $t\left(\mathcal{Q H}_{l=1}\left(\mathbf{C P}^{n}\right)\right)$ decompose as the direct sum

$$
t\left(\mathcal{Q H}_{l=1}\left(\mathbf{C P}^{n}\right)\right)=j\left(\mathbf{C P}^{n}\right) \oplus t(\tau),
$$

and the complete bundle is

$$
\mathcal{Q} \mathcal{H}_{l=1}\left(\mathbf{C P}^{n}\right)=T\left(\mathbf{C P}^{n}\right) \oplus \tau .
$$

We have so far assumed that $l=1$. The case $l=0$ corresponds to the trivial line bundle while, for $l=-1$, the previous construction holds throughout if we replace every bundle with its dual. Thus, on the chart $\mathcal{U}_{k}, k=1, \ldots, n+1$, the dual fibre is the linear span of

$$
{ }_{l=1}\langle 0|, \quad l=1\langle 0| A_{i}(k), \quad i=1,2, \ldots, n .
$$

The case $|l|>1$ will be treated in section 2.3

\subsection{Diagonalisation of the projective Hamiltonian}

Deleting from $\mathbf{C P}{ }^{n}$ the $\mathbf{C P}^{n-1}$-hyperplane at infinity produces the noncompact space $\mathbf{C}^{n}$, which is the classical phase space of the $n$-dimensional harmonic oscillator (now no longer projective, but linear). The corresponding Hilbert space $\mathcal{H}$ is infinitedimensional because the symplectic volume of $\mathbf{C}^{n}$ is infinite.

The deletion of the hyperplane at infinity may be understood from the viewpoint of the Kähler potential corresponding to the Fubini-Study metric on $\mathbf{C P}^{n}$. On the chart $\left(\mathcal{U}_{k}, z_{(k)}\right)$ the Kähler potential reads

$$
K\left(z_{(k)}^{j}, \bar{z}_{(k)}^{j}\right)=\log \left(1+\sum_{j=1}^{n} z_{(k)}^{j} \bar{z}_{(k)}^{j}\right) .
$$

No longer being able to pass holomorphically from a point at finite distance to a point at infinity implies that, on the conjugate chart $\left(\mathcal{U}_{k}, z_{(k)}\right)$, the squared modulus $\left|z_{(k)}\right|^{2}$ is always small and we can Taylor-expand eqn. (5) as

$$
\log \left(1+\sum_{j=1}^{n} z_{(k)}^{j} \bar{z}_{(k)}^{j}\right) \simeq \sum_{j=1}^{n} z_{(k)}^{j} \bar{z}_{(k)}^{j} .
$$

The right-hand side of eqn. (6) is the Kähler potential for the usual Hermitean metric on $\mathbf{C}^{n}$. As such, $\sum_{j=1}^{n} z_{(k)}^{j} \bar{z}_{(k)}^{j}$ equals the classical Hamiltonian for the $n$-dimensional linear harmonic oscillator. Observers on this coordinate chart effectively see $\mathbf{C}^{n}$ as 
their classical phase space. The corresponding Hilbert space is the (closure of the) linear span of the states $\left|m_{1}, \ldots, m_{n}\right\rangle$, where

$$
H_{\operatorname{lin}}\left|m_{1}, \ldots, m_{n}\right\rangle=\sum_{j=1}^{n}\left(m_{j}+\frac{1}{2}\right)\left|m_{1}, \ldots, m_{n}\right\rangle, \quad m_{j}=0,1,2, \ldots,
$$

and

$$
H_{\text {lin }}=\sum_{j=1}^{n}\left(A_{j}^{\dagger}(k) A_{j}(k)+\frac{1}{2}\right)
$$

is the quantum Hamiltonian operator corresponding to the classical Hamiltonian function on the right-hand side of eqn. (6). Then the stationary Schrödinger equation for the projective oscillator reads

$$
H_{\text {proj }}\left|m_{1}, \ldots, m_{n}\right\rangle=\log \left(1+\sum_{j=1}^{n}\left(m_{j}+\frac{1}{2}\right)\right)\left|m_{1}, \ldots, m_{n}\right\rangle,
$$

where

$$
H_{\text {proj }}=\log \left(1+\sum_{j=1}^{n}\left(A_{j}^{\dagger}(k) A_{j}(k)+\frac{1}{2}\right)\right)
$$

is the quantum Hamiltonian operator corresponding to the classical Hamiltonian function on the left-hand side of eqn. (6).

The same states $\left|m_{1}, \ldots, m_{n}\right\rangle$ that diagonalise $H_{\text {lin }}$ also diagonalise $H_{\text {proj }}$. However, eqns. (7)-(10) above in fact only hold locally on the chart $\mathcal{U}_{k}$, which does not cover all of $\mathbf{C P}^{n}$. Bearing in mind that there is one hyperplane at infinity with respect to this chart, we conclude that the arguments of section 2.1 apply in order to ensure that the projective oscillator only has $n$ excited states. Then the occupation numbers $m_{j}$ are either all 0 (for the vacuum state)

$$
|0\rangle_{l=1}=\left|m_{1}=0, \ldots, m_{n}=0\right\rangle,
$$

or all zero but for one of them, where $m_{i}=1$ (for the excited states)

$$
A_{i}(j)^{\dagger}|0\rangle_{l=1}=\left|m_{1}=0, \ldots, m_{i}=1, \ldots, m_{n}=0\right\rangle, \quad i=1, \ldots, n,
$$

and $\operatorname{dim} \mathcal{H}=n+1$ as it should.

One further conclusion that we can draw from the Hamiltonian analysis is that the vacuum $|0\rangle_{l=1}$ of $H_{\text {proj }}$ is nondegenerate. This is so because the $H_{\text {lin }}$ in eqn. (8) has a nondegenerate vacuum, and the logarithm in eqn. (10) is a monotonically increasing function. Therefore the parameter space for physically inequivalent vacua is correctly given by the Picard group. Indeed the latter classifies inequivalent line bundles. This conclusion might appear unnecessary, since we know from the textbooks that the ground state is nondegenerate. However the nondegeneracy of the vacuum of $H_{\text {proj }}$ on $\mathbf{C P}^{n}$ was by no means guaranteed, as the standard proof of nondegeneracy of the vacuum goes back to a mathematical theorem applicable to second-order differential operators [11]. Our Hamiltonian $H_{\text {proj }}$ is not second order. 


\subsection{Construction of the bundle $\mathcal{Q H}(\rho(l), l)\left(\mathbf{C P}^{n}\right)$}

In section 2.1 we have constructed a bundle of $(n+1)$-dimensional Hilbert spaces for the Picard class $l=1$. Now eqns. (2), (3) imply that this vector bundle has $S U(n) \times U(1)$ as its structure group, of which the $(n+1)$-dimensional representation eqn. (1) provides the defining representation (and eqn. (4) its dual).

Any representation $\rho$ of $S U(n+1) \supset S U(n) \times U(1)$ restricts to a representation of $S U(n)$, that we continue to denote by $\rho$. We could pick any such representation, plus a Picard class $l \in \mathbf{Z}$, to construct a $\mathcal{Q H}$-bundle

$$
\mathcal{Q H}(\rho, l)\left(\mathbf{C P}^{n}\right)=\rho\left(T \mathbf{C P}^{n}\right) \oplus \tau^{l} .
$$

Under coordinate changes on $\mathbf{C P}^{n}$, the vacuum $|0\rangle_{l}$ transforms with $t^{l}(\tau)$, while its excitations transform according to jacobian matrices $j_{\rho}\left(\mathbf{C P}^{n}\right)$ expressed in the representation $\rho$. Corresponding to eqn. (13) we would have the transition functions

$$
t\left(\mathcal{Q H}(\rho, l)\left(\mathbf{C P}^{n}\right)\right)=j_{\rho}\left(\mathbf{C P}^{n}\right) \oplus t^{l}(\tau) .
$$

In principle, eqns. (13) and (14) would provide the most general $\mathcal{Q H}$-bundles that one can consider on $\mathbf{C P}^{n}$, were it not for the following reason. In our framework, tangent vectors are quantum states obtained as excitations of the vacuum. As such, a tangent vector in representation $\rho$ cannot be completely arbitrary. It must reflect the fact that it is the result of acting on the vacuum $|0\rangle_{l}$ with a creation operator $A^{\dagger}$. This requirement imposes some constraints on the representation: $\rho$ must be a function of the Picard class $l$ determining the vacuum $|0\rangle_{l}$. The precise dependence $\rho=\rho(l)$ has been obtained in ref. [4], and will be summarised next following an alternative, though equivalent, argument.

Replacing $\mathbf{C P}^{n}$ in section 2.2 with $\mathbf{C P}^{n+l}$, where $l>1$ is the Picard class under consideration on $\mathbf{C P}^{n}$, the vacuum corresponding to the Picard class $l^{\prime}=1$ on $\mathbf{C P}^{n+l}$ has $n+l$ zero occupation numbers,

$$
|0\rangle_{l^{\prime}=1}=\left|m_{1}=0, \ldots, m_{n+l}=0\right\rangle .
$$

On $\mathbf{C P}^{n+l}$ there are $n+l$ creation operators $A_{i}^{\dagger}, i=1, \ldots, n+l$. Picking $n$ out of these and acting with them on $|0\rangle_{l^{\prime}=1}$ we obtain one state that, as seen from $\mathbf{C P}^{n+l}$, is excited. However, as seen from $\mathbf{C P}^{n}$, that same state is either the vacuum $|0\rangle_{l}$ or one of its excitations. There are $\left(\begin{array}{c}n+l \\ n\end{array}\right)$ such independent states. Such is the dimension of the space of states on $\mathbf{C P}^{n}$ corresponding to the Picard class $l>1$.

$S U(n+l)$ Young tableaux with a single column of $n$ boxes correspond to representations of $S U(n+l)$ with dimension $\left(\begin{array}{c}n+l \\ n\end{array}\right)$. By restriction they are also representations of $S U(n+1)$ and, ultimately, of $S U(n)$. Such are the allowed representations $\rho$; as advanced earlier, they depend on the Picard class $l$. It should be borne in mind that the states in these representations are not of the form $A_{i}^{\dagger}|0\rangle_{l}$, with $A_{i}^{\dagger}$, $i=1, \ldots, n$, a creation operator on $\mathbf{C P}^{n}$. Rather, they are of the form $A_{i}^{\dagger}|0\rangle_{l^{\prime}=1}$, with $A_{i}^{\dagger}, i=1, \ldots, n+l$, a creation operator on $\mathbf{C P}^{n+l}$. Thus the vacuum $|0\rangle_{l}, l>1$, is actually degenerate on $\mathbf{C P}^{n}$, since it arises as an excited state with respect to the vacuum 
$|0\rangle_{l^{\prime}=1}$ on $\mathbf{C P}^{n+l}$. However, even if $|0\rangle_{l}, l>1$, is a degenerate vacuum, it continues to hold that $\operatorname{Pic}\left(\mathbf{C P}^{n}\right)=\mathbf{Z}$ is the parameter space for physically inequivalent vacua.

As a consistency check, setting $l=1$ reproduces the dimension $n+1$ of the defining representation. With these conditions on $\rho$, eqns. (13) and (14) are finally correct.

\section{Discussion}

We have argued that the geometry of classical phase space $\mathcal{C}$ determines the possible duality symmetries of the corresponding quantum mechanics. To this end we have introduced the Picard group $\operatorname{Pic}(\mathcal{C})$ as the parameter space for physically inequivalent vacua. This allows to construct different, inequivalent families of Hilbert-space bundles of quantum states over $\mathcal{C}$. In particular, quantum states other than the vacuum appear as tangent vectors to $\mathcal{C}$.

Our analysis has dealt with the case when $\mathcal{C}=\mathbf{C P}^{n}$, for which we have given a classification of all possible $\mathcal{Q H}$-bundles. A mathematical pendulum with an $(n+1)-$ dimensional configuration space has a reduced phase space given by $\mathbf{C P}^{n}$, where the reduction consists in using the energy (a constant of the motion) to decrease the number of degrees of freedom by one [9]. We have also given in section 2.2 another example of a classical dynamics on $\mathbf{C P}^{n}$.

As a complex manifold, $\mathbf{C P}^{n}$ admits a Hermitian metric, so having tangent vectors as quantum states suggests using the Hermitian connection and the corresponding curvature tensor to measure flatness. The freedom in having different nonflat Hilbertspace bundles over $\mathbf{C P}^{n}$ resides in the different possible choices for the complex line bundle $N_{l}\left(\mathbf{C P}^{n}\right)$. Every choice of a vacuum leads to a different set of excitations and thus to a different quantum mechanics. Moreover, the $\mathcal{Q H}$-bundles constructed here are nonflat. This implies that, even after fixing a vacuum, there is still room for duality transformations between different observers on classical phase space. These two facts provide an explicit implementation of quantum-mechanical dualities.

\section{Acknowledgements}

It is a great pleasure to thank J. A. de Azcárraga for encouragement and support. This work has been partially supported by research grant BFM2002-03681 from Ministerio de Ciencia y Tecnología and EU FEDER funds.

\section{References}

[1] C. Vafa, hep-th/9702201

[2] P. Holland, The Quantum Theory of Motion, Cambridge University Press, Cambridge (1993).

[3] J.M. Isidro, Phys. Lett. A301 (2002) 210.

[4] J.M. Isidro, hep-th/0304175, hep-th/0304235

[5] A. Faraggi and M. Matone, Phys. Lett. A249 (1998) 180;

G. Bertoldi, A. Faraggi and M. Matone, Class. Quant. Grav. 17 (2000) 3965. 
[6] E. Davis and G. Ghandour, Phys. Lett. A278 (2001) 239.

[7] J. Anandan, Int. J. Theor. Phys. 41 (2002) 199; quant-ph/0304109

[8] M. Schlichenmaier, An Introduction to Riemann Surfaces, Algebraic Curves and Moduli Spaces, Springer Lecture Notes in Physics 322, Berlin (1989).

[9] V. Arnold, Mathematical Methods of Classical Mechanics, Springer, Berlin (1989).

[10] J. de Azcárraga and J. Izquierdo, Lie Groups, Lie Algebras, Cohomology and some Applications in Physics, Cambridge University Press, Cambridge (1995).

[11] R. Courant and D. Hilbert, Methods of Mathematical Physics, I, Interscience Publishers, New York (1953). 